\section{Hier steht eine Anzeige.}

Springer

\title{
Donepezil: Verbesserung der Kognition nach Bestrahlung
}

Bei Tumorerkrankungen des ZNS ist die Strahlentherapie für eine lokale Tumorkontrolle meist wesentlich, auch wenn sie bei vielen Patienten zur Beeinträchtigung der kognitiven Fähigkeiten führt. Profitieren Betroffene von einer Therapie mit Donepezil?

— twa 50-80\% der Patienten, die sich — aufgrund maligner Prozesse im ZNS einer ionisierenden Bestrahlung unterziehen, entwickeln Nebenwirkungen, die die kognitive Funktion und die Lebensqualität beeinträchtigen. In einer "Open-Label-Phase-II-Studie“ hatte der reversible Inhibitor der Acetylcholinesterase Donepezil zu einer signifikanten Verbesserung der Kognition geführt. Dem gingen Stephen R. Rapp und Kollegen nun in einer randomisierten, placebokontrollierten Studie nach.

Sie behandelten insgesamt 198 erwachsene Patienten mit Hirntumoren (66\%) oder Hirnmetastasen (27\%) mehr als 6 Monate nach Teil- oder Ganzhirnbestrahlung randomisiert entweder mit Donepezil (5 mg täglich über 6 Wochen, dann $10 \mathrm{mg}$ täglich über 18 Wochen) oder Placebo. Die kognitiven Fähigkeiten wurden vor Studienbeginn sowie nach 12 und nach 24 Wochen mit verschiedenen Tests beurteilt.

Im Hinblick auf die allgemeine kognitive Funktion zeigte sich nach 24 Wochen kein Unterschied in beiden Armen $(p=0,48)$. Jedoch sprachen Verbesserungen in spezifischen Funktionsbereichen für eine Donepezil-Therapie, darunter Gedächtnisfunktionen (Wiedererkennung: $\mathrm{p}=0,027$ und Diskrimination: $\mathrm{p}=0,007)$ und motorische Geschicklichkeit $(\mathrm{p}=0,16)$. Zudem schienen Patienten mit größerer primärer Beeinträchtigung eher von Donepezil zu profitieren.

Fazit: Auch wenn Donepezil den kognitiven Gesamtscore nicht signifikant verbesserte, führte die Therapie zu Verbesserungen in einzelnen kognitiven Funktionstests. Dies sollte, so die Forscher, weitere Studien zu Donezepil oder ähn- lichen Substanzen in dieser Indikation rechtfertigen.

Doris Berger

Rapp SR et al. Donepezil for Irradiated Brain Tumor Survivors: A Phase III Randomized Placebo-Controlled Clinical Trial. J Clin Oncol. 2015; 33(15):1653-9.

\section{Kommentar von Prof. Weller:}

Das zunehmende Bewusstsein für die Notwendigkeit der Identifizierung neuroprotektiver Substanzen oder kognitiv förderlicher Interventionen für bestrahlte oder multimodal behandelte Hirntumorpatienten, die zunehmend länger überleben, ist sehr zu begrüßen. Hier liegt eine der wenigen randomisierten Studien zu diesem Thema vor, die den Einsatz von Donepezil, einem in der Alzheimer-Therapie bekannten procholinergen Medikament, prüfte. Es handelt sich um eine große Studie, aber diese Stärke der Studie wird durch die sehr inhomogene Patientenpopulation relativiert.

Es scheint mäßig sinnvoll, Patienten mit unterschiedlichen Bestrahlungsvolumina und unterschiedlichen Primärerkrankungen in einer gemeinsamen Studie zu analysieren, wenngleich hier keine präferentielle Wirksamkeit der Intervention für eine Subgruppe identifiziert wurde, abgesehen davon, dass stärker beeinträchtigte Patienten eher mehr profitierten. Diese Studie legt jedoch nahe, dass procholinerge Interventionen bei diesen Patienten sinnvoll sein könnten und unterstützt die Forderung, dass in ähnlichen Studien möglichst eine Homogenisierung der Patientenpopulation a priori angestrebt werden sollte.

Des Weiteren ist offen, ob die Substanz hier bezüglich Dosis und Applikationsschema optimal ausgenutzt wurde. Weitere Studien zu diesem Thema, die bisher überwiegend in den USA durchgeführt werden, wären sehr zu begrüßen.

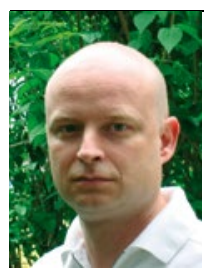

Prof. Dr. med

Michael Weller

Klinik für Neurologie, Universitätsspital Zürich michael.weller@usz.ch 